Finding the link between internalized weight-stigma and binge eating behaviors in Portuguese adult women with overweight and obesity: The mediator role of self-criticism and self-reassurance

Lara Palmeira, José Pinto Gouveia, Marina Cunha, \& Sérgio Carvalho 2017

Eating Behaviors

26: $50-54$

doi: 10.1016/j.eatbeh.2017.01.006 


\title{
Finding the link between internalized weight-stigma and binge eating behaviors in Portuguese adult women with overweight and obesity: The mediator role of self-criticism and self-reassurance
}

\author{
Lara Palmeira, José Pinto Gouveia, Marina Cunha, \& Sérgio Carvalho
}

\begin{abstract}
Literature has emphasized the negative role of internalized self-stigma in unhealthy eating behaviors in individuals with overweight and obesity. Still, less is known about the psychological processes that mediate this relationship. Self-criticism and the inability to reassure the self are considered to play crucial roles on eating behaviors. This study aims to: 1) explore the associations between BMI, weight self-stigma, self-criticism, self-reassurance and binge-eating; 2) explore differences in weight self-stigma and self-criticism tendencies between women with and without Binge Eating Disorder (BED); 3) test the mediator effect of selfcriticism and self-reassurance on the relationship between weight self-stigma and binge-eating severity. Participants were 125 Portuguese adult women with overweight and obesity (MBMI= 34.44; SD =5.51). Participants were weighted and completed a set of self-report measures. BED was assessed through a clinical structured interview. Results showed that women with BED display higher levels of weight self-stigma and self-criticism and lower tendencies to be selfreassuring, than women without BED. Additionally, the mediation model tested indicated that the effect of weight self-stigma on binge eating severity was in part partially mediated by a selfto-self-relationship that is critical, harsh and punitive and by a decreased ability to be reassuring when facing setbacks. Overall, the model accounted for $45 \%$ of binge eating symptoms severity. These findings may inform clinical practice with clients who present high levels of weight selfstigma. Results suggest the relevance of targeting self-criticism and promoting a self-to-self relationship that is caring and reassuring, as a way of diminishing binge eating behaviors.
\end{abstract}

Key-words: Weight self-stigma; Obesity; Binge-eating; self-criticism and self-reassurance; Path analysis 


\section{Highlights}

- Weight self-stigma was positively correlated with self-criticism and binge eating.

- Weight self-stigma was negatively associated with self-reassurance.

- Significant differences between women with and without BED were found.

- Self-criticism and self-reassurance are relevant processes in binge eating symptoms.

- Clinical interventions should focus on the development of self-compassion skills. 


\section{Introduction}

Weight stigma refers to discriminatory attitudes towards people with overweight and obesity, and has a negative impact on the life of individuals with overweight and obesity (e.g. Puhl \& Heuer, 2010). Even in the absence of perceived discrimination, the perception of being a target of stigma is related to poorer health (e.g., Ratner, Halim, \& Amodio, 2013). Moreover, weight stigma is associated with disordered eating behaviors (O’Brien et al., 2016; Vartanian \& Porter, 2016), particularly binge eating (Ashmore, Friedman, Reichmann, \& Musante, 2008; Lillis, Hayes, \& Levin, 2011). Additionally, this weight bias discrimination tends to be internalized by individuals with overweight and obesity reflecting negative self-evaluations of one's weight and physical appearance and perceived discrimination (Durso \& Latner, 2008; Lillis, Luoma, Levin, \& Hayes, 2010). In turn, this is linked with negative health outcomes not exclusively attributed to obesity itself (Latner, Durso, \& Mond, 2013). Weight self-stigma is correlated with psychological distress and disordered eating (e.g. Durso et al., 2012; Pearl \& Puhl, 2014; Schvey \& White, 2015) and affects the quality-of-life of individuals with overweight and obesity (Farhangi, Emam-Alizadeh, Hamedi, \& Jahangiry, 2016; Latner, Barile, Durso, \& O’Brien, 2014; Palmeira, Pinto-Gouveia, \& Cunha, 2016a).

Empirical evidence suggests that individuals who internalize weight-based stereotypes are prone to engage in binge eating (Puhl, Moss-Racusin, \& Schwartz, 2007). In fact, eating is a common way for individuals with overweight and obesity to cope with weight-related discrimination and stigmatization experiences (Puhl \& Brownell, 2006). Additionally, individuals with overweight and obesity with binge eating present poorer health outcomes and quality-of-life than those without binge eating (see Baiano et al., 2014 for a meta-analysis). Specifically, individuals with overweight and obesity who binge eat have higher levels of eating psychopathology, greater medical and psychiatric morbidity (e.g. Bulik, Sullivan, \& Kendler, 2002; Herbozo, Schaefer, \& Thompson, 2015), and present more ineffective strategies of emotional regulation (e.g. Gianini, White, \& Masheb, 2013).

As a result of weight self-stigma, individuals may engage in critical and punitive internal dialogues towards the self, i.e., self-criticism (Gilbert, Clarke, Hempel, Miles, \& Irons, 2004; Gilbert \& Irons, 2005). Self-criticism has been conceptualized as a harsh and punitive way of self-to-self relating, particularly in the face of setbacks or when things go wrong (Gilbert et al., 2004). Although it may be rooted in a desire to self-improve and self-correct, this strategy usually backfires, given that self-criticism focuses on and emphasizes one's flaws and feelings of inferiority (Gilbert et al., 2004; Gilbert \& Irons, 2005). Unintentionally, this may lead to increased negative affect, which is a well-known predictor of binge eating episodes (Gianini et al., 2013). On the other hand, the more toxic and harsh form of self-criticism involves feelings 
of aversion and contempt towards the self (Gilbert et al., 2004; Gilbert \& Irons, 2005). This desire to persecute and punish the self and has been consistently related to more severe forms of psychological suffering (e.g., Castilho, Pinto-Gouveia \& Duarte, 2015). A recent study showed that harsh self-criticism mediates the relationship between body-image shame and binge eating in a sample of 329 non-overweight women (general population and college students) (Duarte, Pinto-Gouveia, \& Ferreira, 2014). In fact, although a few studies have shed some light on how self-criticism and binge eating are related, little is known about the impact of self-criticism, specifically in individuals with overweight and obesity. A study conducted in a sample of patients with eating disorders found that self-criticism predicted depressive symptoms and shape and weight over-evaluation (Dunkley \& Grilo, 2007). Another study, in a sample of 170 patients with binge eating disorder, found that self-criticism was an important mediator between emotional abuse and body dissatisfaction and depression (Dunkley, Masheb, \& Grilo, 2010). Nevertheless, although the role of self-criticism in eating disorders has been growingly unveiled (Goss \& Allan, 2014), little is known about its contribution to the impact of weight self-stigma in binge eating symptoms in people with obesity and overweight.

In contrast to self-criticism, being self-kind, self-compassionate and able to reassure the self when things go wrong seem to be crucial psychological processes that protect against disordered eating (see Braun, Park, \& Gorin, 2016, for a review). Some evidence suggests that self-compassion negatively predicts eating disorders symptoms (Geller, Srikameswaran, \& Zelichowska, 2015; Kelly, Vimalakanthan, \& Carter, 2014; Taylor, Daiss, \& Krietsch, 2015). Nevertheless, it is noteworthy that these studies were conducted in samples from the general population or college students, and not individuals with overweight or obesity.

Compassion-based approaches to eating disorders have been recently developed (Goss \& Allen, 2014) and seem to be effective in reducing binge eating (Kelly \& Carter, 2015). These approaches specifically aim to promote the development of a self-to-self- relationship characterized by a kind, caring and supportive attitude, instead of being critical, punitive and harsh towards oneself (Gilbert et al., 2014; Neff, 2003). In fact, some evidence points out that when people with overweight and obesity experience setbacks, they tend to become self-critical and have difficulty in being self-compassionate. In turn, this is linked with struggles in maintaining healthy behaviors (e.g., Gilbert et al., 2014). Thus, this calls for the importance of exploring the role of self-reassurance in binge eating in patients with overweight and obesity. Additionally, studies about how self-compassion relates to weight self-stigma and binge eating are still scant, with only one study showing that self-compassion mediated the impact of weight self-stigma on mental and physical health outcomes (Hilbert, Braehler, Schmidt, Löwe, Häuser, \& Zenger, 2015). 
The current study has three aims: 1) to study the associations between weight selfstigma, self-criticism, self-reassurance and binge-eating in a sample of women with overweight or obesity with and without Binge Eating Disorder (BED). We expect positive and moderate to strong correlations between weight self-stigma, hated and inadequate-self and binge eating symptoms. Conversely, moderate to strong negative associations are expected between the above mentioned variables and reassured-self; 2) to explore differences between binge-eaters and non-binge-eaters in weight self-stigma, self-criticism and self-reassurance. We expect that women with BED present higher levels of weight self-stigma and self-criticism and lower selfreassuring abilities than those without BED. The third goal was to test whether self-criticism and self-reassurance mediate the relationship between weight self-stigma and binge eating symptoms.

\section{Methods}

\subsection{Participants}

From the 134 Portuguese women with overweight or obesity seeking nutritional treatment in Coimbra invited to participate, nine declined. A sample of 125 participants were interviewed by experienced clinical psychologists using the Portuguese versions of Eating Disorder Examination Interview (Ferreira, Pinto-Gouveia, \& Duarte, in preparation) and the appendix H from Structured Clinical Interview for DSM-IV TR (SCID; translated by Maia, 2006) to determine the existence of Binge Eating Disorder. BED diagnosis was established following the criteria from DSM-V. According to DSM-V, 54 participants (43.2\%) presented BED and 73 participants did not. Participants' mean age was $41.14(S D=8.72)$, with an average of $14.96(S D=3.15)$ years of education. Sample's average Body Mass Index (BMI) was 34.44 $(S D=5.51)$. Concerning marital status, $62.4 \%$ were married and $20.8 \%$ were single. The majority came from low to medium socio-economic status (87.21\%).

\subsection{Procedures}

Prior to data collection, the current study was approved by the ethics committee of Coimbra's University Hospital (CHUC) and by the scientific committee of the Psychology Faculty of University of Coimbra, Portugal. Participants were invited to participate by a research team member on the day of their nutritional appointment. Participants were informed about the voluntary and confidential nature of the collaboration, as well as the main study's 
goals. After signing a written informed consent, participants were screened for BED individually and completed the self-report measures. The questionnaires took approximately 20 min to be completed.

\subsection{Measures}

Demographic Data. Participants' age, years of education and current height were selfreported.

BMI. Participants were weighted with their clothes (without shoes) using the same Body Composition Analyzer (Tanita TBF-300) accurate to $0.1 \mathrm{~kg}$.

Weight self-stigma Questionnaire (WSSQ; Lillis et al., 2010; Palmeira, Cunha, \& PintoGouveia, 2016b) includes 12 items that assess weight self-stigma in people with overweight and obesity. It measures negative thoughts and emotions about being overweight and fear of enacted stigma. Participants are asked to rate each item in a 5-point Likert scale $(1=$ strongly disagree; 5 $=$ strongly agree). Higher scores indicate higher levels of weight self-stigma. Both the original $(\alpha=0.88)$ and Portuguese $(\alpha=0.85)$ versions presented good psychometric properties (Lillis et al., 2010; Palmeira et al., 2016b).

Forms of Self-Criticizing/Attacking \& Self-Reassuring Scale (FSCRS; Gilbert et al., 2004; Castilho et al., 2015) is 22-item self-report measure with three subscales: inadequate-self (focused on personal inadequacies and feelings of inferiority, "There is a part of me that feels I am not good enough"), hated-self (focused on condemning and attacking the self; "I have a sense of disgust with myself') and reassured-self (focused on being warm and in comforting the self; "I am gentle and supportive with myself"). It measures the tendency to criticize or reassure the self when facing failures or errors. Items are rated on a 5-point scale $(0=$ "Not at all like me" to 4 = "Extremely like me"). Both the original and Portuguese versions revealed good internal consistencies in clinical and non-clinical samples ranging from 0.83 to 0.91 (Castilho et al., 2015; Gilbert et al., 2004).

Binge Eating Scale (BES; Gormally et al., 1982; Duarte et al., 2015) consist of 16 items. Participants are asked to choose which of the given statements best describes their experience concerning binge-eating symptoms. Scale's scores range from 0 to 46, with higher scores reflecting higher severity of binge eating symptoms. Both the original and Portuguese versions showed high internal consistency $(\alpha=0.88)$.

Table 1 displays the internal consistency for all variables in study. 
Table 1 Pearson moment correlation on all variables for the total sample $(\mathrm{N}=125)$.

\begin{tabular}{lccccccccr}
\hline Measures & $M$ & $S D$ & $\alpha$ & 1 & 2 & 3 & 4 & 5 & 6 \\
\hline 1. BMI & 34.44 & 5.51 & - & - & & & & \\
2. Binge eating & 21.06 & 9.67 & 0.89 & 0.04 & - & & & \\
3. Weight self-stigma & 41.50 & 9.37 & 0.91 & 0.04 & $0.62^{* * *}$ & - & & & \\
4. Hated self & 5.19 & 3.54 & 0.65 & 0.16 & $0.49^{* * *}$ & $0.50^{* * *}$ & - & & \\
5. Inadequate self & 19.06 & 7.35 & 0.87 & -0.03 & $0.42^{* * *}$ & $0.60^{* * *}$ & $0.68^{* * *}$ & - & \\
6. Reassured self & 16.79 & 6.64 & 0.89 & -0.12 & $-0.52^{* * * *}$ & $-0.55^{* * *}$ & $-0.52^{* * *}$ & $-0.42^{* * *}$ & - \\
\hline
\end{tabular}

Note. $* * * p<0.001 ;$ BMI $=$ Body Mass Index.

\subsection{Data analysis}

Data analyses were performed using IBM SPSS Statistics 20 and AMOS software. Preliminary data analyses were performed to explore the adequacy of the data. Pearson correlation coefficients were conducted to examine the associations between BMI, WSSQ, hated, inadequate and reassured-self and BES (Cohen, Cohen, West, \& Aiken, 2003). To explore differences between binge eaters and non-binge eaters in all variables, independent sample $t$ tests and Cohen's d effect size were calculated (Field, 2013). Cohen's cutoff values were followed: Cohen's d between 0.2 and 0.4 - small effects; between 0.5 and 0.7 - medium effects and above 0.8 - large effects (1988 cited in Tabachnick \& Fidell, 2007). Finally, to test whether self-criticism and self-reassurance mediated the relationship between weight selfstigma (WSSQ) and binge eating symptomatology (BES), while controlling for BMI, a path analysis was used. Path analysis allows the simultaneous examination of structural relationships, as well as the examination of direct and indirect paths (e.g., Schumacker \& Lomax, 2004). The Maximum Likelihood method was chosen because it allows for the estimation of all model path coefficients and to compute fit statistics. To assess the model fit we relied on several goodnessof-fit measures and recommended cut-points (Kline, 2005): Chi-Square ( $\chi 2)$, Normed ChiSquare ( $\chi 2 /$ d.f.), Comparative Fit Index (CFI $\geq 0.95$, desirable; Hu \& Bentler, 1998), TuckerLewis Index (TLI $\geq 0.95$, desirable; Hu \& Bentler, 1998), Goodness of Fit Index (GFI $\geq 0.95$, desirable; Jöreskog \& Sörbom, 1996), Root Mean Square Error of Approximation (RMSEA $\leq$ 0.05 , good fit; Kline, 2005) with a 95\% confidence interval. The bootstrap with 2000 resamples and $95 \%$ bias-corrected confidence interval was used to analyze the mediational effects. The effect is considered statistically significant at $p<0.05$ if the interval between the lower and the upper bound does not include zero (Kline, 2005). 


\section{Results}

\subsection{Preliminary Data Analyses}

All variables presented acceptable values of skewness and kurtosis (SK $<|3|$ and $\mathrm{Ku}<$ $|8-10|)$ which indicate no severe bias to normal distribution. Multicollinearity was not found (VIF < 5; Kline, 2005). Also, the analysis of multivariate outliers using Mahalanobis distance statistic $\left(D^{2}\right)$ did not detect the presence of any outlier. There were no missing data.

\subsection{Correlation analysis}

Table 1 displays the means, standard deviations and Pearson's correlation coefficients for all variables in study. As can be seen, BMI was not significantly related to any variable. WSSQ showed moderate and positive associations with hated-self, inadequate-self and BES. In turn, reassured-self revealed negative and moderate associations with WSSQ, BES, hated and inadequate-self.

\subsection{Differences between binge eaters and non-binge eaters}

Table 2 depicts the means, standard deviations, $t$-test differences and Cohen's $d$ for all variables for the two groups (with and without BED). Results reveal that binge eaters presented significantly higher levels of binge eating symptoms, weight self-stigma, hated and inadequate self, and lower levels of reassured-self when compared with non-binge eaters. Differences regarding binge eating symptoms, and weight self-stigma reflect very large effects, whereas differences in hated, inadequate and reassured-self reflect small effect sizes (Table 2). Additionally, no statistically significant differences were found regarding BMI. 
Table 2. Means (M), standard deviations (SD), t-test differences and Cohen's d effect size for all variables in both groups $(\mathrm{N}=125)$.

\begin{tabular}{lcccccccc}
\hline & $\begin{array}{c}\text { With BED } \\
(\mathrm{n}=54)\end{array}$ & \multicolumn{2}{c}{$\begin{array}{c}\text { Without BED } \\
(\mathrm{n}=71)\end{array}$} & & & \\
\hline & $M$ & $S D$ & $M$ & $S D$ & $t(d f)$ & $p$ & $\begin{array}{c}\text { Cohen's } \\
\mathrm{d}\end{array}$ \\
\hline BMI & 34.70 & 5.95 & 34.24 & 5.19 & $0.455(123)$ & 0.650 & 0.08 \\
Binge eating & 28.42 & 7.34 & 15.45 & 7.14 & $9.943(123)$ & $<0.001$ & 1.80 \\
Weight self-stigma & 46.06 & 7.52 & 38.02 & 6.93 & $5.227(123)$ & $<0.001$ & 0.94 \\
Hated self & 6.11 & 3.65 & 4.50 & 3.32 & $2.555(123)$ & 0.012 & 0.46 \\
Inadequate self & 20.98 & 7.52 & 17.63 & 6.93 & $2.564(123)$ & 0.012 & 0.46 \\
Reassured self & 13.74 & 6.51 & 19.07 & 5.80 & -4.806 & $(123)$ & $<0.001$ & 0.47 \\
\hline
\end{tabular}

\subsection{Path analysis}

A path analysis was conducted to test the mediator effect of self-criticism (both hated and inadequate-self dimensions) and self-reassurance on the relationship between weight selfstigma and binge eating symptoms severity, while controlling BMI. The initial model was tested through a fully saturated model with 20 parameters. Model fit indices were not reported as fully saturated models have a perfect model fit. The analysis of the path coefficients from the fully saturated model revealed four non-significant direct paths, namely: the path from BMI $\rightarrow$ BES $(t$ statistics $=-0.072 ; p=0.550) ; \mathrm{BMI} \rightarrow$ inadequate-self $(t$ statistics $=-0.062 ; p=0.515)$; inadequate-self $\rightarrow$ BES ( $t$ statistics $=-0.097 ; p=0.459)$; BMI $\rightarrow$ reassured-self $(t$ statistics $=-$ $0.137 ; p=0.122$ ) and were progressively removed. The final model (Fig. 1) included all the remaining statistically significant path coefficients. The model presented an excellent model fit: $\chi^{2}(4, N=125)=3.699, p=0.448 ; \chi^{2} /$ d.f. $=0.925 ; \mathrm{GFI}=0.990 ; \mathrm{CFI}=1.000 ; \mathrm{TLI}=1.000 ;$ RMSEA $=0.000,[C I=0.000 ; 0.131] ; p=0.596$. 


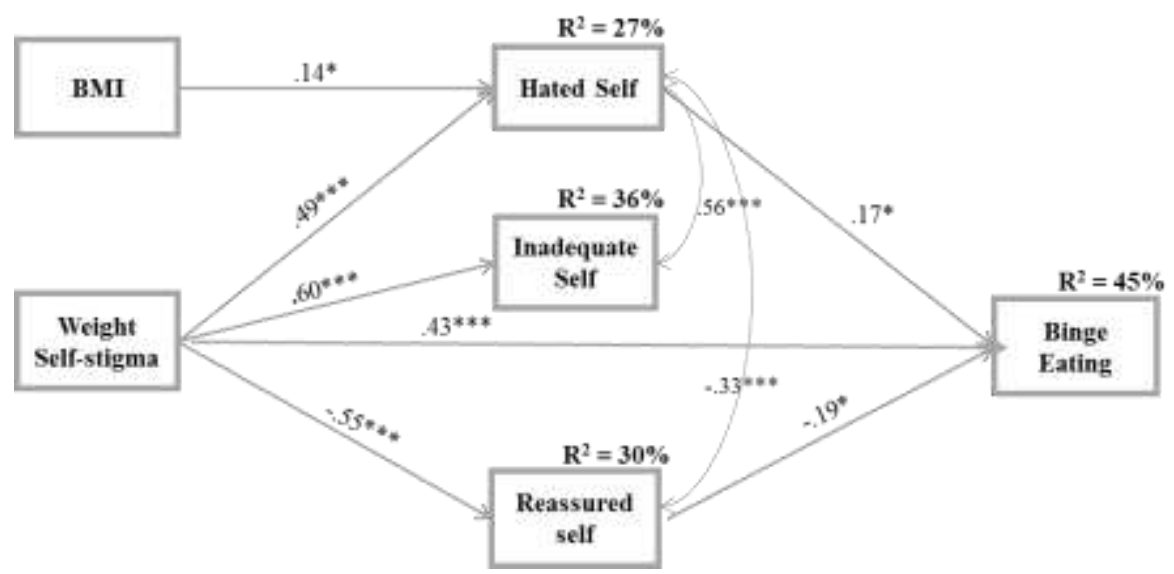

Fig. 1. Final Path Model $(\mathrm{N}=125)$.

Note. ${ }^{*} p<0.05 ;{ }^{* * *} p<0.001$; only significant standardized path coefficients among variables are presented.

\subsection{Mediation analysis}

Bootstrap with 2000 resamples and $95 \%$ bias-corrected confidence interval was used to analyze the total, direct and indirect effects. Results revealed that weight self-stigma showed both direct and indirect effects on BES. The direct effect of WSSQ on BES was $\beta=0.433$ based on $95 \%$ CI: $(0.267 ; 0.579, p=0.001)$ and the indirect effect was $\beta=0.188$ based on $95 \% \mathrm{CI}$ : $(0.086 ; 0.314, p=0.001)$. This indirect effect occurred through hated-self $(b=0.493 \times 0.173=$ $0.085)$ and reassured-self $(b=-0.552 \times-0.187=-0.103)$. Moreover, hated-self $(\beta=0.173$; based on 95\% CI: .005; .330, $p=.043)$ and reassured-self $(\beta=-0.187$; based on 95\% CI: -0.377 ; $0.007, p=0.039)$ also presented significant direct effects on BES. The total effect of WSSQ on BES was $\beta=0.621$ based on $95 \% \mathrm{CI}(0.512 ; 0.719, p=0.001)$, that represent the sum of the standardized direct effect with the standardized indirect effect. Finally, BMI had a significant direct effect on hated-self ( $\beta=0.142$; based on 95\% CI: $0.016 ; 0.268, p=0.026)$ and an indirect effect on BES (through hated-self) of $\beta=0.025$ based on $95 \% \mathrm{CI}(0.001 ; 0.075, p=0.032)$. The final model (Fig. 1) accounted for $27 \%$ of the variance of hated-self, $30 \%$ of reassured-self, $36 \%$ of inadequate-self and $45 \%$ of binge eating symptoms.

\section{Discussion}

Recently, studies have emphasized the damaging impact of internalized weight-stigma in healthy eating behaviors of individuals with overweight and obesity (Ashmore et al., 2008; Pearl \& Puhl, 2014; Schvey \& White, 2015). Nonetheless, the psychological processes through which this relationship is operated on are still underexplored. The current study contributes to 
the existing literature by testing the mediator role of self-criticism and self-reassurance on the relationship between weight self-stigma and binge eating symptoms.

In line with previous studies (e.g., Ashmore et al., 2008; Durso et al., 2012), results from correlation analyses showed that weight self-stigma was positively and significantly associated with binge eating symptoms. As expected, participants with higher levels of weight self-stigma revealed higher self-criticism a less self-reassurance. To best of our knowledge, this is the first study to explore these results, although they corroborate that weight-stigma internalization is related to poor health outcomes (e.g., Ratner et al., 2013). Moreover, these results also seem to align with other studies that found that high self-criticism and low levels of self-compassion are associated with disordered eating behaviors (e.g., Duarte et al., 2014; Dunkley \& Grilo, 2007, Dunkley et al., 2010). Interestingly, BMI was not significantly associated with any variable in study, which may have resulted from the reduced BMI variability from our sample. However, these results are consistent with studies that found that the negative impact of weight self-stigma on disordered eating goes beyond the impact of weight itself (Latner et al., 2013).

In addition, we sought to explore differences between women with and without BED. Contrarily to previous findings (e.g., Palmeira et al., 2016a), no difference across groups were found regarding BMI. Nonetheless, Palmeira et al. (2016a) asserted the presence of binge eating using BES scores, while the current study conducted a structured clinical assessment. Women with BED showed significantly higher levels of weight self-stigma and self-criticism and lower levels of self-reassurance, than women without binge-eating. This is consistent with previous findings that suggest that individuals with BED present poorer health outcomes when compared to those without BED (e.g., Bulik et al., 2002; Durso et al., 2012). Our study adds to the existent literature by showing that women with BED, when compared to women without BED, tend to adopt a more critical attitude towards the self and have lower abilities to reassure the self when facing setbacks or failures.

The major contribution of this study was to explore whether self-criticism and selfreassurance mediated the influence of weight self-stigma on binge eating symptoms, while controlling for BMI. Results suggest that the effect of weight self-stigma on binge eating symptoms partially occurs through a harsh and attacking attitude towards the self (hated-self) and through a decreased ability in reassuring the self when things go wrong. Interestingly, the less toxic and harsh form of self-criticism (inadequate-self) did not mediate this relationship. It seems that women who internalize stigmatizing weight messages tend to develop a self-to-self relationship that is characterized by a harsh and punitive attitude, as well as the inability to 
reassure the self when facing setbacks. In turn, this way of dealing with difficulties and failures seems to be related to higher severity of binge eating symptoms.

Still, it is noteworthy that results also revealed a direct effect of weight self-stigma on binge eating symptoms. This stresses the importance of weight stigma internalization on women's tendency to engage in binge eating. Another possibility is that other unexplored processes might contribute to this relationship. In fact, research has shown that weight-related experiential avoidance is an important process on the relationship between weight self-stigma and quality-of-life (e.g., Lillis et al., 2011; Palmeira et al., 2016a). Finally, BMI also had an indirect effect on binge-eating symptoms, through its effect on hated-self. It seems that presenting a higher BMI is indirectly linked to more binge-eating symptoms due to one's tendency to attack and condemn oneself when facing setbacks.

This study has some limitations: 1) its cross-sectional design, prevents us from inferring causality. Futures studies should consider replicating our findings following a longitudinal design; 2) the sample used comprised only adult women, which does not allow to generalize the results for other genders or age groups. In fact, being BED the most common eating disorder in men it would be worth examining the existence of gender differences on the relationship between weight self-stigma and binge eating; 3) results concerning the hated-self dimension should be interpreted with caution given its low internal consistency; 4) the model tested is limited, as other processes (e.g., decentering abilities, rumination) may be involved in the relationship between weight self-stigma and binge eating symptoms. Still, our goal was to explore the role of self-criticism and self-reassurance in this relationship. Future studies should explore our findings using a multi-group approach (which our sample size did not allow) to test differences between individuals with and without BED.

Nevertheless, the current study has significant strengths: a) our study comprised a specific clinical sample of women with overweight and obesity, rather than a community sample; b) BMI and BED were not self-reported, but assessed through a body composition analyzer and a clinical structured interview, respectively; c) this is the first study to explore the role of weight self-stigma and self-criticism on binge-eating symptoms.

In conclusion, our findings provide evidence that may be useful for clinicians working with women with overweight and obesity, particularly those with BED. Specifically, it suggests that therapists should consider weight self-stigma and the more toxic form of self-criticism (hated-self) as important aspects to tackle in therapy in order to reduce binge eating symptoms. One way to address these aspects is through the development of self-compassion, using a compassionate-based approach such as Compassion Focused Therapy (CFT). In fact, CFT was specifically developed to provide a de-shaming setting, to decrease patients' shame feelings and 
self-criticism, and to help them develop a warmer and more caring self-to-self relationship, particularly when facing difficulties or failure (e.g., Goss \& Allan, 2014). In this context, being compassionate towards oneself seems to provide intrinsic motivation and the ability to maintain healthy behaviors even when setbacks occur (e.g., Neff, Rude, \& Kirkpatrick, 2007). 


\section{References}

Ashmore, J.A., Friedman, K.E, Reichmann, S.K., \& Musante, G.J. (2008). Weight-based stigmatization, psychological distress, \& binge eating behavior among obese treatmentseeking adults. Eating Behaviors, 9(2), 203-209. doi: 10.1016/j.eatbeh.2007.09.006.

Baiano, M., Salvo, P., Righetti, P., Cereser, L., Baldissera, E., Camponogara, I., \& Balestrieri, M. (2014). Exploring health-related quality of life in eating disorders by a cross-sectional study and a comprehensive review. BMC Psychiatry, 14, 165. doi:10.1186/1471-244X14-165

Braun, T.D., Park, C.L., \& Gorin, A. (2016). Self-compassion, body image, and disordered eating: A review of the literature. Body Image, 17, 117-131. doi: 10.1016/j.bodyim.2016.03.003.

Bulik, C.M., Sullivan, P.F., \& Kendler, K.S. (2002). Medical and psychiatric morbidity in Obese women with and without binge eating. International Journal of Eating Disorders, 32 (1), 72-78. doi: 10.1002/eat.10072

Castilho, P., Pinto-Gouveia, J., \& Duarte, J. (2015). Exploring Self-criticism: Confirmatory Factor Analysis of the FSCRS in Clinical and Nonclinical Samples. Clinical Psychology and Psychotherapy, 22(2), 153-164. doi.org/10.1002/cpp.1881.

Cohen, J., Cohen, P., West, S.G., \& Aiken, L.S. (2013). Applied multiple regression/correlation analysis for the behavioral sciences. Routledge: New Jersey

Dunkley, D.M., \& Grilo, C.M. (2007). Self-criticism, low self-esteem, depressive symptoms, and over-evaluation of shape and weight in binge eating disorder patients. Behaviour Research and Therapy, 45(1), 139-149. doi: 10.1016/j.brat.2006.01.017

Dunkley, D.M., Masheb, R.M., \& Grilo, C.M. (2010). Childhood maltreatment, depressive symptoms, and body dissatisfaction in patients with binge eating disorder: The mediating role of self-criticism. International Journal of Eating Disorders, 43(3), 274-281. doi: 10.1002/eat.20796

Durso, L.E., \& Latner, J.D. (2008). Understanding self-directed stigma: Development of the weight bias internalization scale. Obesity Journal, 16(2), 80-86. doi: 10.1038/oby.2008.448.

Durso, L.E., Latner, J.D., White, M.A., Masheb, R.M., Blomquist, K.K., Morgan, P.T., \& Grilo, C.M. (2012). Internalized weight bias in obese patients with binge eating disorder: Associations with eating disturbances and psychological functioning. International Journal of Eating Disorders, 45:3, 423-427. doi:10.1002/eat.20933 
Duarte, C., Pinto-Gouveia, J., \& Ferreira, C. (2014). Escaping from body image shame and harsh self-criticism: Exploration of underlying mechanisms of binge eating. Eating Behaviors, 15(4), 638-643. doi: 10.1016/j.eatbeh.2014.08.025

Duarte, C., Pinto-Gouveia, J., \& Ferreira, C. (2015). Expanding binge eating assessment: Validity and screening value of the binge eating scale in women from the general population. Eating Behaviors, 18, 41-47 doi:10.1016/j.eatbeh.2015.03.007

Farhangi, M. A., Emam-Alizadeh, M., Hamedi, F., \& Jahangiry, L. (2016). Weight self-stigma and its association with quality of life and psychological distress among overweight and obese women. Eating and Weight Disorders-Studies on Anorexia, Bulimia and Obesity, 1-6. doi:10.1007/s40519-016-0288-2

Field, A. (2013). Discovering statistics using IBM SPSS statistics (4th ed.). LosAngeles, London: SAGE

Geller, J., Srikameswaran, S., \& Zelichowska, J. (2015). Resilience to shape and weight concerns and disordered eating: the role of self-compassion. Advances in Eating Disorders: Theory, Research and Practice, 3(1), 4-12. doi: 10.1080/21662630.2014.945604.

Gianini, L.M., White, M.A., \& Masheb, R.M. (2013). Eating pathology, emotion regulation, and emotional overeating in obese adults with binge eating disorder. Eating Behaviors, 14(3), 309-313. doi:10.1016/j.eatbeh.2013.05.008

Gilbert, J., Stubbs, R.J., Gale, C., Gilbert, P., Dunk, L., \& Thomson, L. (2014). A qualitative study of the understanding and use of 'compassion focused coping strategies' in people who suffer from serious weight difficulties. Journal of Compassionate Health Care, 1(1), 1. doi: 10.1186/s40639-014-0009-5

Gilbert, P., Clarke, M., Hempel, S., Miles, J., \& Irons, C. (2004). Criticizing and reassuring oneself: An exploration of forms, styles and reasons in female students. British Journal of Clinical Psychology, 43, 31-50. doi: 10.1348/014466504772812959

Gilbert, P., \& Irons, C. (2005). Focused therapies and compassionate mind training for shame and self-attacking. In P. Gilbert (Ed.), Compassion: Conceptualisations, research and use in psychotherapy (pp. 263-325). London: Routledge

Goss, K. \& Allan, S. (2014). The development and application of compassion-focused therapy for eating disorders (CFT-E). British Journal of Clinical Psychology, 53(1), 62-77. doi:10.1111/bjc.12039

Gormally, J., Black, S., Daston, S., \& Rardin, D. (1982). The assessment of binge eating severity among obese persons. Addictive Behaviors, 7, 47-55. 
Herbozo, S., Schaefer, L.M., \& Thompson, J.K. (2015). A comparison of eating disorder psychopathology, appearance satisfaction, and self-esteem in overweight and obese women with and without binge eating. Eating Behaviors, 17, 86-89. http://dx.doi.org/10.1016/j.eatbeh.2015.01.007

Hilbert, A., Braehler, E., Schmidt, R., Löwe, B., Häuser, W., \& Zenger, M. (2015). Selfcompassion as a resource in the self-stigma process of overweight and obese individuals. Obesity Facts, 8(5), 293-301. doi: 10.1159/000438681

Hu, L., \& Bentler, P.M. (1998). Fit indices in covariance structure modeling: Sensitivity to underparameterized model misspecification. Psychological Methods, 3, 424-453.

Jöreskog, K.G., \& Sörbom, D. (1996). LISREL 8 user's reference guide. Chicago: Scientific Software International.

Kelly, A.C., \& Carter, J.C. (2015). Self-compassion training for binge eating disorder: A pilot randomized controlled trial. Psychology and psychotherapy: Theory, research and practice, 88(3), 285-303. doi: 10.1111/papt.12044

Kelly, A.C., Vimalakanthan, K., \& Carter, J.C. (2014). Understanding the roles of self-esteem, self-compassion, and fear of self-compassion in eating disorder pathology: An examination of female students and eating disorder patients. Eating Behaviors, 15(3), 388-391. doi: 10.1016/j.eatbeh.2014.04.008

Kline, R.B. (2005). Principles and Practice of Structural Equation Modeling (2nd ed.). 366, New York: Guilford.

Latner, J.D., Barile, J.P., Durso, L.E., \& O’Brien, K.S. (2014). Weight and health-related quality of life: The moderating role of weight discrimination and internalized weight bias. Eating Behaviors, 15, 586-590. doi:10.1016/j.eatbeh.2014.08.014

Latner, J. D., Durso, L. E., \& Mond, J. M. (2013). Health and health-related quality-of-life among treatment-seeking overweight and obese adults: Associations with internalized weight bias. Journal of Eating Disorders, 1(3). doi:10.1186/2050-2974-1-3.

Lillis, J., Hayes, S., \& Levin, M. (2011). Binge eating and weight control: The role of experiential avoidance. Behavior Modification, 35, 3252-3264. doi: $10.1177 / 0145445510397178$.

Lillis, J., Luoma, J., Levin, M., \& Hayes, S. (2010). Measuring weight self-stigma: The weight self-Stigma questionnaire. Obesity, 18(5), 971-976. doi:10.1038/oby.2009.353.

Neff, K.D. (2003). The development and validation of a scale to measure self-compassion. Self and Identity, 2(3), 223-250. doi: 10.1080/15298860309027 
Neff, K.D., Kirkpatrick, K.L., \& Rude, S.S. (2007). Self-compassion and adaptive psychological functioning. Journal of research in personality, 41(1), 139-154. doi:10.1016/j.jrp.2006.03.004

O'Brien, K.S., Latner, J.D., Puhl, R.M., Vartanian, L.R., Giles, C., Griva, K., \& Carter, A. (2016). The relationship between weight stigma and eating behavior is explained by weight bias internalization and psychological distress. Appetite, 102(1), 70-76. Doi: 10.1016/j.appet.2016.02.032

Palmeira, L., Pinto-Gouveia, J., \& Cunha, M. (2016a). The role of weight self-stigma on the quality of life of women with overweight and obesity: A multi-group comparison between binge eaters and non-binge eaters. Appetite, 105, 782-789. doi: 10.1016/j.appet.2016.07.015.

Palmeira, L., Cunha, M., \& Pinto-Gouveia, J. (2016b). The weight of weight self-stigma in women with overweight and obesity. Manuscript submitted for publication.

Pearl, R.L., \& Puhl, R.M. (2014). Measuring internalized weight attitudes across body weight categories: Validation of the Modified Weight Bias Internalization Scale. Body image, 11(1), 89-92. doi: 10.1016/j.bodyim.2013.09.005

Puhl, R.M., \& Brownell, K.D. (2006). Confronting and coping with weight stigma: An investigation of overweight and obese adults. Obesity, 14(10), 1802-1815. doi: 10.1038/oby.2006.208

Puhl, R., \& Heuer, C.A. (2010). Obesity stigma: Important considerations for public health. American Journal of Public Health, 100, 1019-1028.

Puhl, R.M., Moss-Racusin, C.A., \& Schwartz, M.B. (2007). Internalization of weight bias: Implications for binge eating and emotional well-being. Obesity, 15(1), 19-23. doi: 10.1038/oby.2007.521

Ratner, K.G., Halim, M.L., \& Amodio, D.M. (2013). Perceived stigmatization, ingroup pride, and immune and endocrine activity evidence from a community sample of black and latina Women. Social Psychological and Personality Science, 4(1), 82-91. doi: $10.1177 / 1948550612443715$

Schumacker, R.E., \& Lomax, R.G. (2004). A beginner's guide to structural equation modeling (2nd edition). Mahwah, NJ: Lawrence Erlbaum Associates.

Schvey, N.A., \& White, M.A. (2015). The internalization of weight bias is associated with severe eating pathology among lean individuals. Eating Behaviors, 17, 1-5. doi: 10.1016/j.eatbeh.2014.11.001

Tabachnick, B \& Fidell, L. (2007). Using multivariate statistics. New York: Pearson Education Inc. 
Taylor, M.B., Daiss, S., \& Krietsch, K. (2015). Associations among self-compassion, mindful eating, eating disorder symptomatology, and body mass index in college students. Translational Issues in Psychological Science, 1(3), 229. doi: 10.1037/tps0000035

Vartanian, L.R., \& Porter, A.M. (2016). Weight stigma and eating behavior: A review of the literature. Appetite, 102, 3-14. doi: 10.1016/j.appet.2016.01.034 